\title{
IMPLEMENTATION OF A TOOL FOR CONTROL STRUCTURE ASSESSMENT
}

\author{
Mikael Petersson* \\ ABB Automation Technology Products, SE-205 22 Malmö, Sweden \\ Karl-Erik Årzén, Henrik Sandberg, Lena de Maré \\ Department of Automatic Control, Lund Institute of Technology \\ PO Box 118, SE-221 00 Lund, Sweden
}

\begin{abstract}
A method for assessing disturbances in SISO loops has been developed. The method indicates if the SISO loop under consideration will benefit from an addition of feedforward control from a measured disturbance. An implementation in Java of the methodology is presented in the paper.
\end{abstract}

Keywords: Process Control, PID Control, Supervision, Control Structure Selection, Feedforward Control, Java

\section{INTRODUCTION}

The process industry of today is highly automated. The control loops outnumber by far the number of operators and people in the maintenance and instrumentation departments. The processes are getting more complex, but the control problems are still attacked with mostly PIcontrollers. In industry, processes often change over time, e.g. by modification of different process sections. Unfortunately, these changes do not always propagate correctly to the control system, resulting in performance loss.

During the last decade much research has been devoted to monitoring of control loop performance using normal operating data (Harris, 1989; Desborough and Harris, 1993; Kozub and Garcia, 1993; Stanfelj et al., 1993; Hägglund, 1995; Hägglund, 1999; Horch and Isaksson, 1998; Harris et al., 1999). With this in mind, a tool for analysing the control loop structure based on measurements has been developed. The goal is to incorporate this tool in an environment for control loop performance and structure assessment. Such a tool could be used to first determine whether the control loops are well tuned or not, and then to verify if an ap-

\footnotetext{
* The author is an industrial PhD-student at the Department of Automatic Control, Lund Institute of Technology. E-mail: mikael.petersson@se.abb.com
}

propriate control strategy has been chosen, and, when not, to get a measure on how much better control performance that can be achieved using another control strategy.

The paper describes the first steps toward such a tool. A tool for control structure assessment has been implemented in Java. The tool is able to evaluate if feedforward control action should be added to a SISO loop. The signals of the loop and one or several extra measurable signals are required to perform the assessment. By analysing the transient responses the tool evaluates the influence of the extra signals. The result is presented as an index. The indices of different loops can be compared in order to focus the maintenance people on the control loops which offer most improvement.

In the paper the research approach is first presented. The method is described in Section 3 along with examples. Then the implementation environment is presented. The results from laboratory experiments are discussed in Section 5 , ending with the conclusion in Section 6.

\section{APPROACH}

The starting point is a SISO system and an additional measurable signal, $x$ (see Figure 1). This additional signal may, for example, be an 


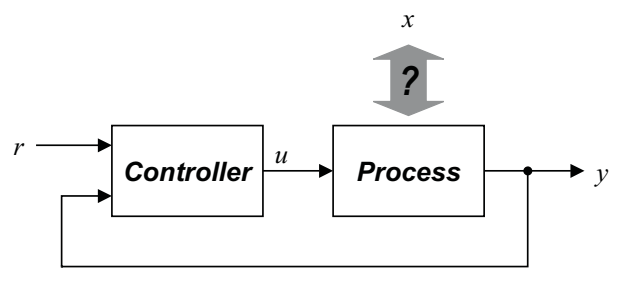

Fig. 1 A SISO system and an additional signal

additive disturbance, but it may also be an output from the process. The problem is to decide if the signal has a relation to the loop, the nature of this relation, and if the signal can be used to improve the performance of the loop. Each possible control structure has its own consideration during the analysis of the additional signal. The feedforward case was treated in (Petersson et al., 2001) and this paper deals with the implementation of those results and the questions that arise during an implementation.

\section{THE FEEDFORWARD INDEX}

The index implemented evaluates the influence of a measurable additional signal on a SISO control loop. The additional signal is considered to be an additive disturbance, and the controller is considered to be tuned. The calculated index gives an idea of the relation between the time constant in the disturbance path and the time constant of the process path.

\subsection{Definition}

The basic idea is to compare the controller's response to a disturbance, $u(t)$, with two reference responses. The references are the controller's response to the same disturbance entering before and after the process respectively, i.e. the two extreme entry points of a disturbance in the control loop. The two control reference responses are denoted $u_{\text {before }}(t)$ and $u_{\text {after }}(t)$.

The area in between the two reference responses constitutes the reference area. The index is calculated by dividing the disturbance area, between the after-reference and the response due to the measured disturbance, with the reference area, see Figure 2. The index will thus have a value larger than zero, and close to one if the average residence times (Åström and Hägglund, 1995; Marlin, 1995) of the disturbance and process paths are approximately the same. If the average residence time of the disturbance path is larger the index will be larger than one.

An index close to or larger than one indicates a signal that enters before or early in the process,

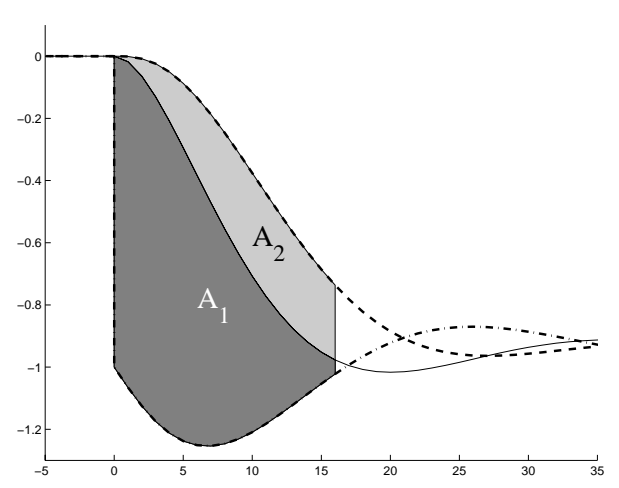

Fig. 2 Comparison of control signals. The response to the disturbance is shown in solid, the after-reference is dash-dotted, and the before-reference is dashed. The area generated by the disturbance is labelled $A_{1}$, and the reference area is the sum of the two areas, $A_{1}+A_{2}$. The feedforward index is the ratio between $A_{1}$ and the reference area.

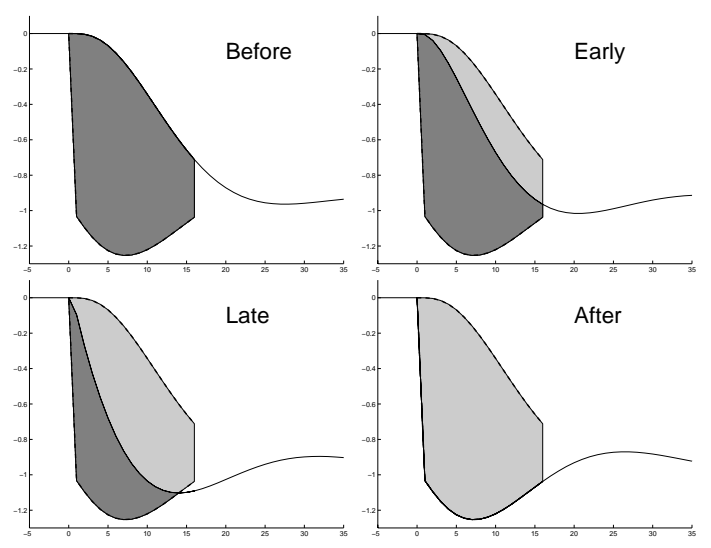

Fig. 3 The effect of a step disturbance for the different entry points in the process (as indicated in the figures). The indices were calculated to $1.0,0.74,0.36$ and 0.0 , in the order from before to after.

and that it would improve the performance of the loop if a feedforward control action was added. Disturbances receiving indices close to zero are considered to enter late in the process and they are best handled by feedback control.

The effect of a unit step disturbance on a process is shown in Figure 3. The process consists of three first-order filters, each with a time constant of 5 and unit gain. The disturbance entry points are before the process, between the first and second filter, between the second and third filter, and after the process.

\subsection{Calculation}

To calculate the index the signals need to be scaled (Petersson et al., 2001). The scaling is depending on the gains of the process, the size of the measured disturbance, $\Delta_{\text {dist }}$, and the size of the disturbance used to generate the reference, 
$\Delta_{r e f}$. Let the static gain of the transfer functions from the controller output to the process output and the gain from the disturbance to the process output be denoted by $P(0)$ and $D(0)$, respectively. The scaled measured control output is then defined as $\bar{u}(t)=P(0) \Delta_{\text {ref }} /\left(D(0) \Delta_{\text {dist }}\right) u(t)$ and the scaled after-reference as $\bar{u}_{\text {after }}(t)=$ $P(0) u_{\text {after }}(t)$. The index is now given by the following equation

$$
\eta_{\mathrm{FF}}=\frac{\int_{0}^{T_{\mathrm{ar}}}\left(\bar{u}(t)-\bar{u}_{\mathrm{after}}(t)\right) d t}{\int_{0}^{T_{\mathrm{ar}}}\left(u_{\mathrm{before}}(t)-\bar{u}_{\mathrm{after}}(t)\right) d t}
$$

where $T_{\text {ar }}$ is the average residence time, which for a first-order plus deadtime model equals the sum of the deadtime and the time-constant.

\subsection{Reference generation}

The references can be generated in at least three ways. The first case involves generation of the references experimentally from unit step disturbances, $\theta$. The disturbances are introduced in the controller, $C$, before and after the process, $P$, see Figure 4 . The responses can be expressed by the following equations: $u_{\text {before }}=$ $-C P /(1+C P) \theta$ and $u_{a f t e r}=-C /(1+C P) \theta$. Once a disturbance, $d$, and the control signal $u_{d}$ have been measured a transfer function from a fictive step to the measured disturbance is estimated, $d=D \theta$. The transfer function $D$ is used to transform the references before calculating the index.

$$
\begin{aligned}
u_{\text {before }}^{D} & =D u_{\text {before }}=D \frac{-C P}{1+C P} \theta \\
& =\frac{-C P}{1+C P} d
\end{aligned}
$$

Similar result exists for the transformation of the after-reference $u_{a f t e r}^{D}=D u_{a f t e r}$.

While the transformation of the shape of the disturbance is a linear transformation, the change of controller parameters is non-linear. Therefore it is assumed that the controller will have a fixed operating point. If this is not the case, new

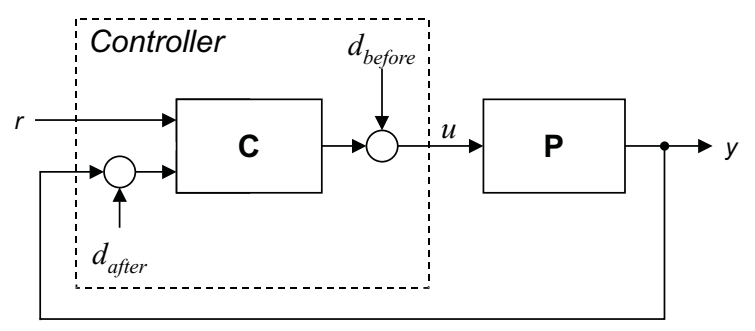

Fig. 4 Schematic view over generation of reference signal by introducing disturbances to signals in the controller references must be recorded once the controller is tuned at the new operating point.

The second approach consists of performing experiments on the process when a disturbance has been measured. The measured disturbance is played back to the process in order to obtain the references. This approach is not likely to be accepted in industry since it upsets the process during the evaluation phase.

The third way requires a simple process model, for example a first-order plus deadtime model, describing the relationship between the controller output and the process output. The controller parameters and its structure are considered known. The references are then generated by simulations with the measured disturbance as input.

In the experiments presented in Section 5 the references were obtained through experiments at the same operating point where the disturbance occurred.

\section{IMPLEMENTATION}

The index has been implemented, together with a PID-controller and supporting functions, in Java. The software environment and the laboratory process are presented below.

The use of Java enables development in a mixed environment; Linux in the laboratory, Sun Solaris 8 in the offices, and Windows 2000 when out of the office. Different classes have been developed locally to support the implementation of a control system in Java.

In the development phase a virtual process package, which is developed at the department, has been used. The virtual process package makes it possible to run the controller with either the real process or a simulated process.

The simulated process, which includes an animated graphical user interface, can be approximated in many different ways, for example Euler forward, Tustin, or discrete time. In Figure 5 the animated graphics of a tank process is shown. To start the software together with the virtual process an argument is added to the command, which forces the I/O's to use the virtual process instead of the hardware devices.

The main classes of the implementation are the controller and the index classes. The controller and the index-calculation run in separate threads. The index thread subscribes to data from the controller in order to get correct time synchronisation of the process data. The index calculation is event triggered, from the index thread, once sufficient information is available. 

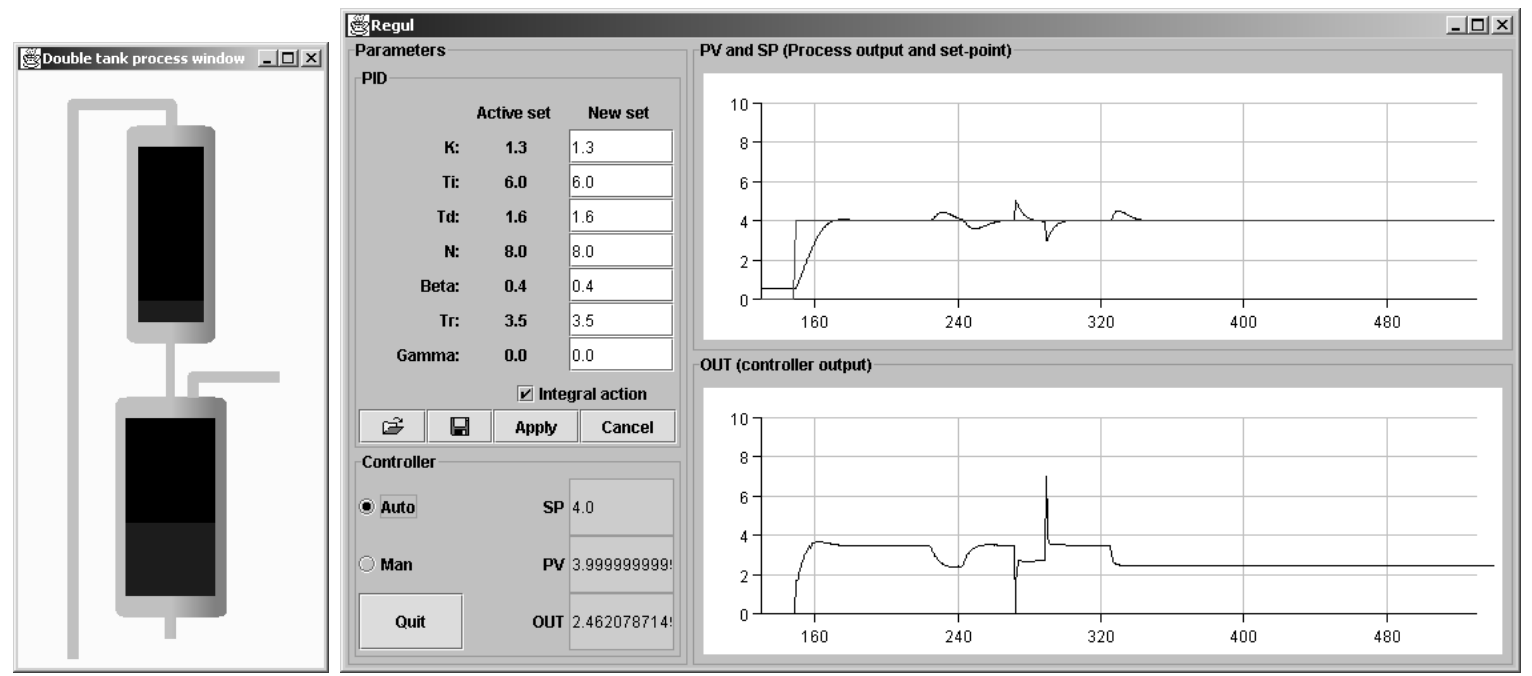

Fig. 5 The animated Java Swing interface for a tank process and the controller user interface. The user is able to edit, save and load controller parameters in the left pane. The lower left pane handles the controller mode, set-point source, and manual output value.

The references are manually generated by introducing step disturbances in the control loop. The user interface for the assessment is shown in Figure 6.

\section{EXPERIMENTS}

This section presents the results from the experiments performed on the laboratory equipment of the Department of Automatic Control in Lund.

We have used the quadruple tank (Johansson, 2000), see Figure 7. The process has one pump for each column and there are valves that enable the flow to enter the lower tank of the other column as well. The main process consists of an upper and a lower tank. The disturbance path is just a pump connected to the main process' lower tank. There is thus no extra dynamics in the disturbance path, and the time constant of the disturbance path is smaller or equal to the one of the process. An index between one and zero can therefore be expected. A typical outcome of an assessment of a disturbance is shown in Figure 8.

\subsection{Disturbance assessment}

When calculating the index controller responses from a disturbance together with two references are needed. In Figure 8 the control signals for the three phases of the assessment are shown. Extracting the controller responses, and normalising the time, reveal the raw data for calculating the index (see Figure 9).

Two process parameters are needed for the cal-

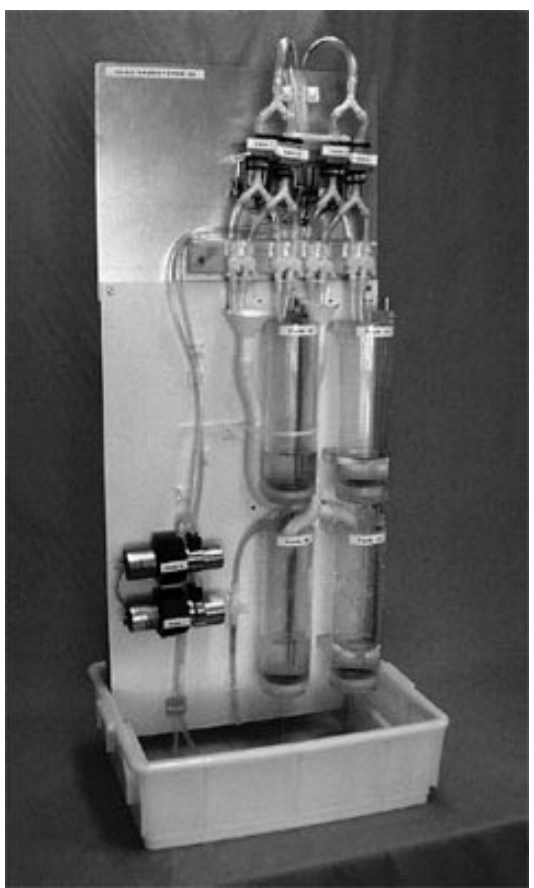

Fig. 7 The quadruple tank process

culation of the index. The estimation of the gain from the controller output to the process output, and from the disturbance to the process output was done by performing two step experiments, see Figure 10. The estimation can also be done with ordinary closed-loop identification methods. The result of scaling the signals of Figure 9 is shown in Figure 11. The index was calculated to 0.91 , indicating that feedforward may be useful. 


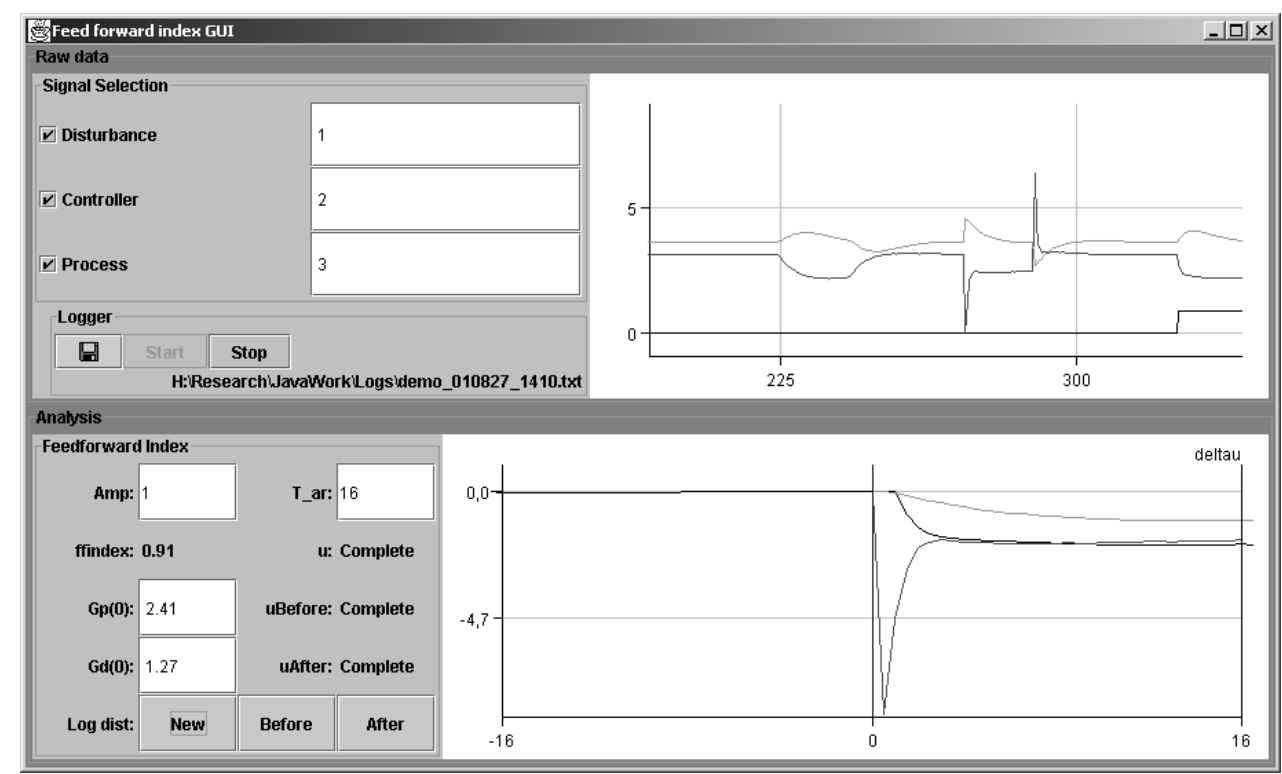

Fig. 6 The feedforward index interface. This prototype has two logger areas. The upper shows the true signals and the lower shows the control response of an disturbance together with the references responses. The left pane offers possibility to save the data from the experiment to a log, signal selection, process parameters, controls for obtaining index data, and index output
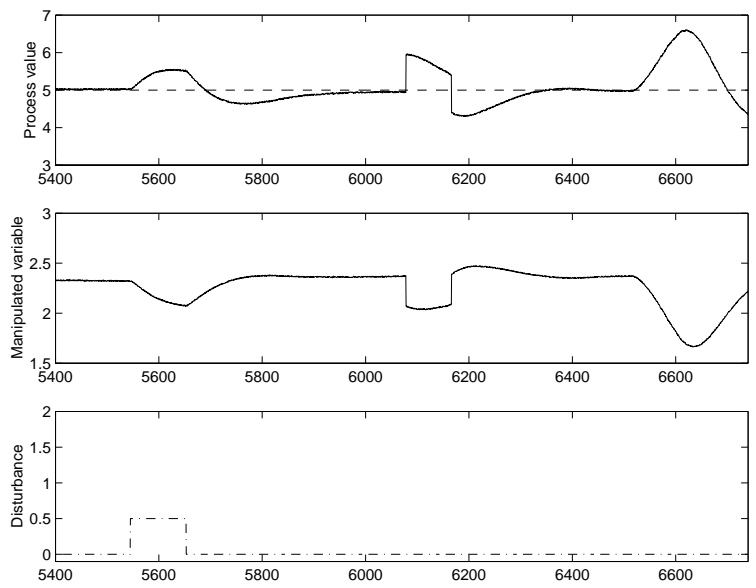

Fig. 8 Measurements from an experiment on the laboratory tank process. The signals show, in time, first a measured disturbance and the effect it has on the loop. Then the two references are generated starting with the reference where the disturbance enters after the process.

\subsection{Implementation issues}

For controllers with derivative action, like the PID, the after reference generates a spike which may lead to a saturated control signal. In Figure 6 this can be seen both in the signal logger and the index plot. Saturations will affect the integral part of the controller. If the saturation only lasts for some samples the effect on the index is negligible if the operating point is sufficiently far from the saturating limit.

Processes can have different dynamics in different directions, for example a tank with a larger

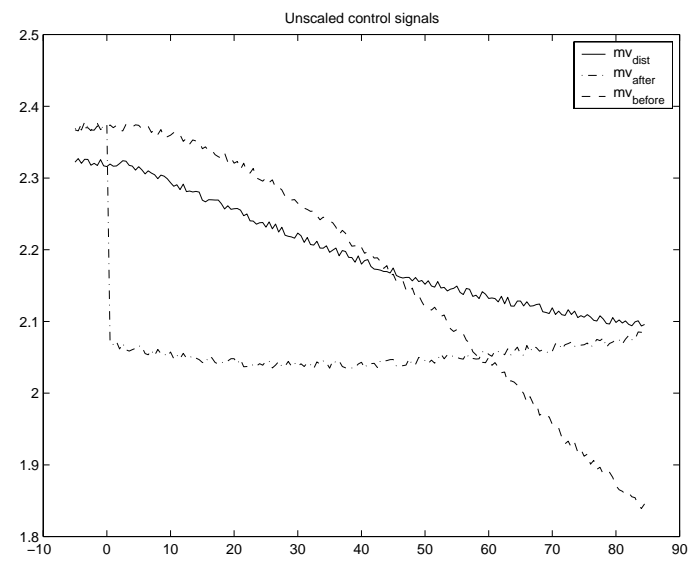

Fig. 9 Control responses for index calculation before scaling and detrending. The signals which will be scaled are the response to the disturbance (solid) and the afterreference (dash-dotted).

inflow than outflow capacity. This imposes the need for two sets of references depending on the direction of the disturbance.

The index-calculation will fail in some circumstances. The process should be in steady state when a reference or disturbance is recorded. An ongoing reference generation must be aborted if the set-point is changed. This is also valid when another disturbance than the one under evaluation upsets the process. 

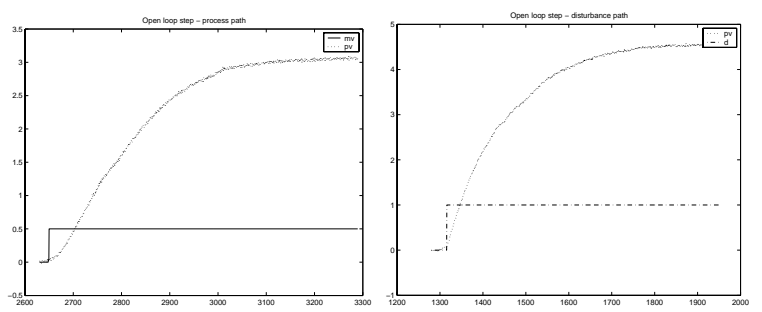

Fig. 10 Open loop step responses. Left figure shows a step in process input, and the right a step in the disturbance

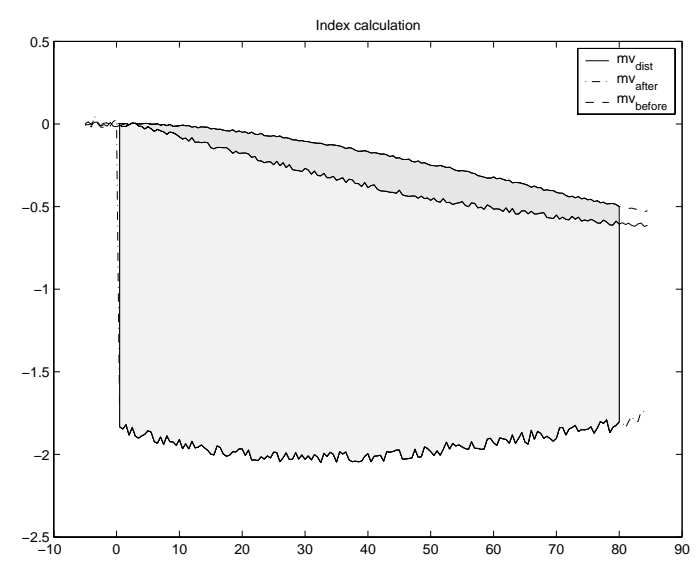

Fig. 11 Control responses after scaling, with index-areas indicated. The index is 0.91 .

\section{CONCLUSIONS}

Incorporating the feedforward index in a control structure tool makes it possible to focus the efforts of the maintenance staff on the most needing loops. The tool can be used to supervise several loops in combination with signals that are possible disturbances sources. The tool then presents the pairing of loop and signals together with an index indicating which signal that can be used for extra feedforward control actions.

The future work is focused on automating the methodology. The implementation in an industrial control system demands safety logic to get safe operation and appropriate results.

There is need for detection methods in order to detect a disturbance, change of set-points, and interfering disturbances. The latter concerns the gathering of data for the assessment, which must be aborted if another disturbance affects the loop.

There is also a need for identification of, at least, the gain of the process and the gain of the disturbance path. This includes estimation of the level of excitation of the signals in order to get proper estimates.

There is then the question of mode and state changes of the controller, for example tuning of the controller parameters, which influences the assessment. Further on, the dialog with the operator must be as simple as possible, but there is also a demand for information so that the person conducting the assessment can trust the results.

\section{REFERENCES}

Åström, K. J. and T. Hägglund (1995): PID Controllers: Theory, Design and Tuning, second edition. Instrument Society of America, Research Triangle Park, NC.

Desborough, L. and T. Harris (1993): "Performance assessment measures for univariate feedforward/feedback control." The Canadian Journal of Chemical Engineering, 71, August, pp. 605-616.

Hägglund, T. (1995): "A control-loop performance monitor." Control Engineering Practice, 3:11, pp. 1543-1551.

Hägglund, T. (1999): "Automatic detection of sluggish control loops." Control Engineering Practice, 7, pp. 1505-1511.

Harris, T., C. Seppala, and L. Desborough (1999): "A review of performance monitoring and assessment techniques for univariate and multivariate control systems." $J$. of Process Control, 9, pp. 1-17.

Harris, T. J. (1989): "Assessment of control loop performance." Canadian Journal of Chemical Engineering, 67, pp. 856-861.

Horch, A. and A. J. Isaksson (1998): "A modified index for control performance assessment." In American Control Conference. Philadelphia.

Johansson, K. H. (2000): "The quadruple-tank process-a multivariable laboratory process with an adjustable zero." IEEE Transactions on Control Systems Technology, 8:3.

Kozub, D. and C. Garcia (1993): "Monitoring and diagnosis of automated controllers in chemical process industries." In AIChE meeting. St Louis.

Marlin, T. (1995): Process Control, Designing Processes and Control Systems for Dynamic Performance. McGraw Hill.

Petersson, M., K.-E. Årzén, and T. Hägglund (2001): "Assessing measurements for feedforward control." In de Carvalho, Ed., European Control Conference - ECC'01, pp. 432437. Porto, Portugal.

Stanfelj, N., T. E. Marlin, and J. F. MacGregor (1993): "Monitoring and diagnosing process control performance: The single-loop case." Ind. Eng. Chem. Res., 32:2, pp. 301-314. 